1 Universidade Federal da Bahia (UFBA), Escola de Enfermagem - Salvador (BA), Brasil.

ednirassis@hotmail.com

2 Universidade Federal da Bahia (UFBA), Instituto de Humanidades, Artes e Ciências (IHAC) e Instituto de Saúde Coletiva (ISC), Programa de

Pós-graduação em Saúde Coletiva - Salvador (BA), Brasil.

carment@ufba.br

3 Universidade Federal da Bahia (UFBA), Instituto de Saúde Coletiva (ISC) Salvador (BA), Brasil. marilucejbv@yahoo.com.br

\section{Análise da produção científica nacional sobre o trabalho da enfermeira (1988-2014)}

\author{
Analysis of the national scientific production on the work of nurses \\ (1988-2014)
}

Ednir Assis Souza', Carmen Fontes Teixeiraㄹ, Mariluce Karla Bomfim de Souza ${ }^{\mathbf{2}}$

RESUMO O estudo visa analisar a produção científica sobre o trabalho da enfermeira no Brasil, no período de 1988 a 2014. A base de dados utilizada foi a Scientific Electronic Library Online (SciELO). Selecionaram-se 222 artigos, caracterizados segundo o ano de publicação, periódico, abordagem teórico-metodológica e área temática, buscando-se identificar mudanças no trabalho da enfermeira. Os resultados apontam a introdução de novos conhecimentos e tecnologias, com perda de autonomia, bem como deterioração das relações interpessoais, sofrimento e sobrecarga de trabalho. Discute-se a possível correlação desses processos com as mudanças na organização e gestão dos sistemas de saúde no Brasil.

PALAVRAS-CHAVE Trabalho. Cuidados em enfermagem. Enfermeiras e enfermeiros.

ABSTRACT The study aims to analyze the scientific production on the work of nurse in Brazil, from 1988 to 2014. The database used was the Scientific Electronic Library Online (SciELO). We selected 222 articles, characterized by the year of publication, journal, theoretical-methodological approach and thematic area, seeking to identify changes in the work of the nurse. The results point to the introduction of new knowledges and technologies, with loss of autonomy, as well as deterioration of interpersonal relationships, suffering and work overload. It discusses the possible correlation of such processes with the changes in the organization and management of health systems in Brazil.

KEYWORDS Work. Nursing care. Nurses. 


\section{Introdução}

O processo de trabalho em saúde é um dos temas que têm merecido a atenção dos pesquisadores do campo da saúde coletiva desde os seus primórdios, nos anos 70 do século passado (DONNANGELO, 1975; MENDES-GONÇALVES, 1994). Uma revisão recente (PINTO ET AL., 2013) dos estudos que abordam esse tema aponta a multiplicidade de objetos e de abordagens, que incluem desde estudos sobre mercado de trabalho e práticas profissionais até estudos sobre gestão do trabalho e educação na saúde. Embora abrangente, a revisão assinalada não se detém em analisar a produção científica sobre o trabalho das diversas categorias profissionais, o que aponta a possibilidade de realização de estudos específicos que deem conta das mudanças que vêm ocorrendo no âmbito das diversas práticas, em função da reorganização dos sistemas de saúde no Brasil (PAIM, 2008; PAIM ET AL., 2011).

O trabalho da enfermeira como parte integrante da produção de serviços de saúde tem sido objeto de análise, dado ao contingente expressivo desses profissionais (MACHADO, 2015). Os estudos pioneiros nessa área (MELO, 1986; SILVA, 1987; PIRES, 1989; ALMEIDA; ROCHA, 1997) buscaram caracterizar o trabalho da enfermeira, consoante a divisão técnica e social do trabalho em saúde, especificando o lugar da enfermeira no modo de organização tecnológica do trabalho, sobretudo no modelo biomédico. Nesse sentido, destacou-se o caráter limitante das práticas da enfermeira em realidades concretas e emergiu o questionamento acerca da pertinência das suas práticas, em relação à finalidade a que essas se propõem (ALMEIDA; ROCHA, 1997). Estudos mais contemporâneos privilegiaram aspectos mais interacionais relativos ao trabalho da enfermeira, com destaque para a autonomia (KRAEMER; DUARTE; KAISER, 2011), as relações profissionais e institucionais (URBANETTO; CAPELLA, 2004; CAHÚ ET AL., 2014), além da visão da enfermeira sobre o seu trabalho (VEIGA; FERNANDES; PAIVA, 2011).
Apesar das diferenças de abordagem, constata-se que tais estudos apontam a ocorrência de mudanças impostas ao trabalho da enfermeira pelo conjunto de fatores (econômicos, políticos e sociais) que, ao longo do tempo, têm influenciado os modos de organização e funcionamento dos sistemas e serviços de saúde, nos quais a enfermeira se insere, reconfigurando seu processo de trabalho nas dimensões assistenciais, gerenciais, educativas e de pesquisa. Nesse sentido, cabe perguntar quais as principais mudanças que vêm ocorrendo no processo de trabalho da enfermeira no Brasil, o que pode ser identificado a partir de um estudo que tome como objeto a produção científica sobre esse tema. Assim, este artigo tem por objetivo mapear a produção científica nacional sobre o trabalho da enfermeira no Brasil, entre 1988 e 2014, levando em conta o processo de construção do Sistema Único de Saúde (SUS) e, ao mesmo tempo, a expansão do Sistema de Atenção Médica Supletiva (Sams), os quais constituem o cenário de práticas em que essa profissional se insere.

\section{Material e métodos}

Trata-se de um estudo, de caráter descritivo e exploratório, que analisou estudos relativos à temática 'trabalho da enfermeira' publicados no Brasil, no período de 1988 a 2014. A busca foi feita na biblioteca virtual Scientific Electronic Library Online (SciELO), entre os meses de março e agosto de 2014, haja vista essa base ser a maior provedora de periódicos indexados pelo Diretório de Periódicos de Acesso Aberto - Directory of Open Access Journals (Doaj), agregando a maioria dos periódicos latino-americanos indexados pela Web of Science e Scopus. Os periódicos publicados nacionalmente centram-se, grande parte, em comunidades de pesquisa nacionais e regionais que veiculam pesquisas de interesse local ou com base em metodologias e particularidades relacionadas com 
as comunidades de pesquisa do País (PACKER ET AL., 2014). A seleção dos estudos foi feita de acordo com os seguintes passos:

a) Definição dos descritores: tomou-se como ponto de partida a identificação dos descritores: trabalho, processo de trabalho, cuidado, práticas, enfermeira, enfermeiro. Inicialmente pesquisados na base Descritores em Ciências da Saúde (DeCS) da Bireme (Centro Latino-Americano e do Caribe de Informação em Ciências da Saúde). Entretanto, por não ter sido encontrado o descritor 'processo de trabalho' e pela generalização do descritor 'trabalho', optou-se pelo uso do operador booleano 'and'. Do mesmo modo, para os descritores 'cuidado' e 'práticas', cuja manutenção justifica-se pelo uso comum, respectivamente em referência ao objeto de trabalho e trabalho da enfermeira. Assim, a busca foi feita pela combinação dos descritores, resultando no seguinte quantitativo de resumos: 'processo de trabalho and enfermeira' ( $\mathrm{n}=16)$; 'processo de trabalho and enfermeiro' ( $n=42)$; 'trabalho and enfermeira' ( $n=134)$; 'trabalho and enfermeiro' $(\mathrm{n}=332)$; 'cuidado and enfermeira' ( $\mathrm{n}=139)$; 'cuidado and enfermeiro' ( $\mathrm{n}=307)$; 'práticas and enfermeira' $(\mathrm{n}=39)$ e 'práticas and enfermeiro' $(\mathrm{n}=84)$, totalizando 1.088 artigos, respeitando o recorte temporal.

b) Seleção de resumos: foram considerados critérios de inclusão a publicação dos resumos de artigos em revistas científicas; indexados na base SciELO; e que trataram da temática do trabalho, processo de trabalho, cuidado e práticas da(o) enfermeira(o). Foram excluídos os resumos em duplicidade, estudos com ausência de resumo e impertinência temática, quais sejam: trabalho, processo de trabalho, cuidado e práticas de auxiliares, técnicos em enfermagem e parteiras, trabalho da equipe de saúde, biografias profissionais e formação profissional, sendo, portanto, selecionados um total de 222 artigos (figura 1).

c) Classificação dos resumos selecionados: os 222 resumos identificados foram dispostos em planilha Excel ${ }^{\circledR}$, lidos e classificados de acordo com as seguintes variáveis: ano de publicação, periódico em que foi publicado; área temática; abordagem teórico-metodológica; mudanças identificadas no trabalho da enfermeira.

d) O processamento dessas informações permitiu a elaboração de gráficos e tabelas, utilizando o programa Starta 10, de acordo com as variáveis anteriormente descritas. Desse modo, construiu-se a evolução temporal dos estudos, sua distribuição por periódico, abordagem teórico-metodológicas e área temática, focando-se a análise nas informações relativas às mudanças ocorridas no trabalho da enfermeira no período considerado. 


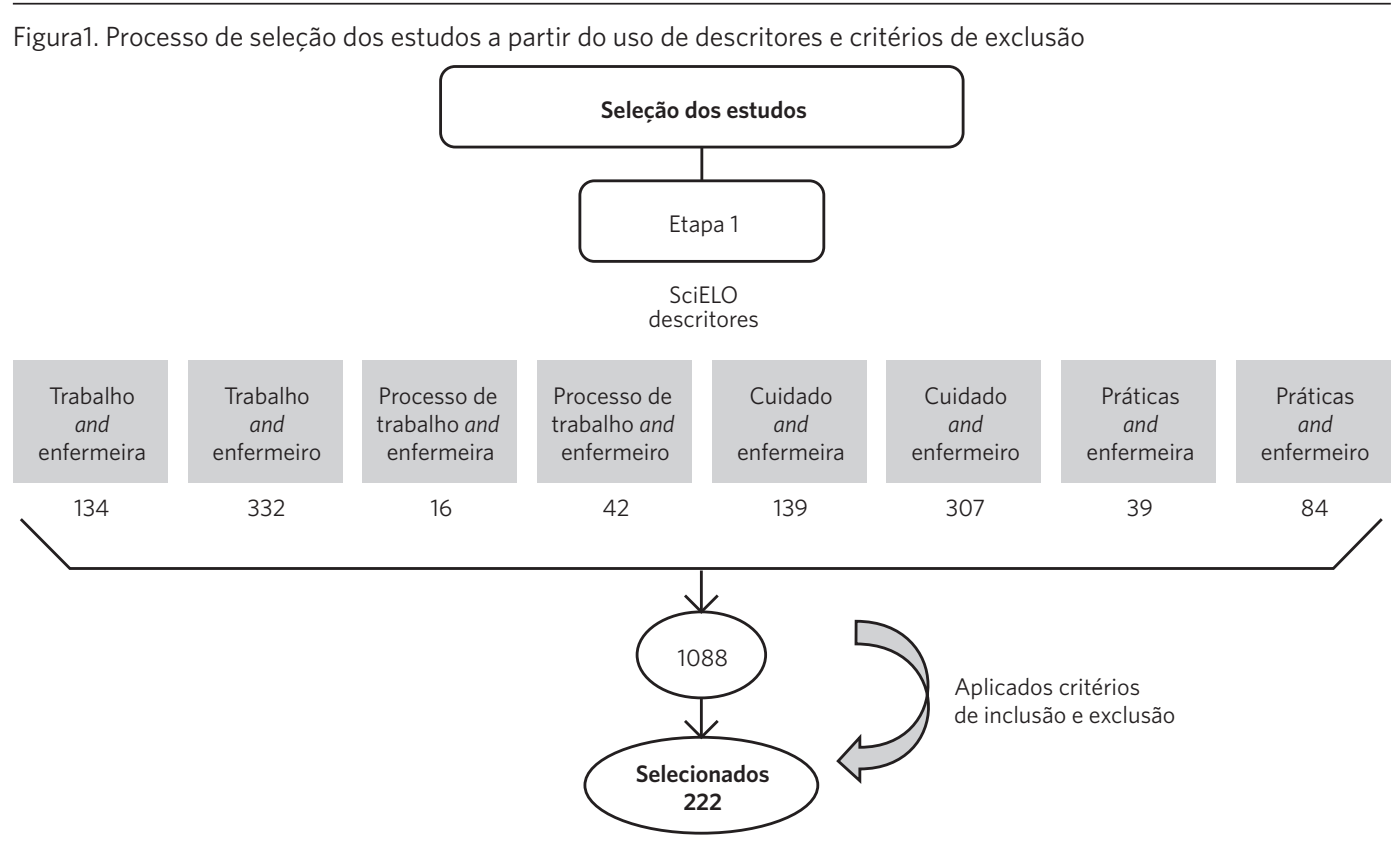

\section{Resultados}

\section{Evolução temporal dos estudos selecionados}

A análise da evolução temporal dos estudos revela, em primeiro lugar, que o registro de publicações sobre o trabalho da enfermeira tem início em 1992, com variação pouco significativa nessa década. A partir dos anos 2000, observa-se uma tendência ao aumento do número de estudos, embora exista certa oscilação entre os anos finais da década. A produção científica da década de 1990 concentrou apenas 9,9\% do total de estudos, enquanto, na década seguinte, o percentual atingiu $51 \%$ do total, observando, ainda, uma elevação do número de estudos entre os anos 2011 e 2013, verificando-se uma queda em 2014 (gráfico 1).

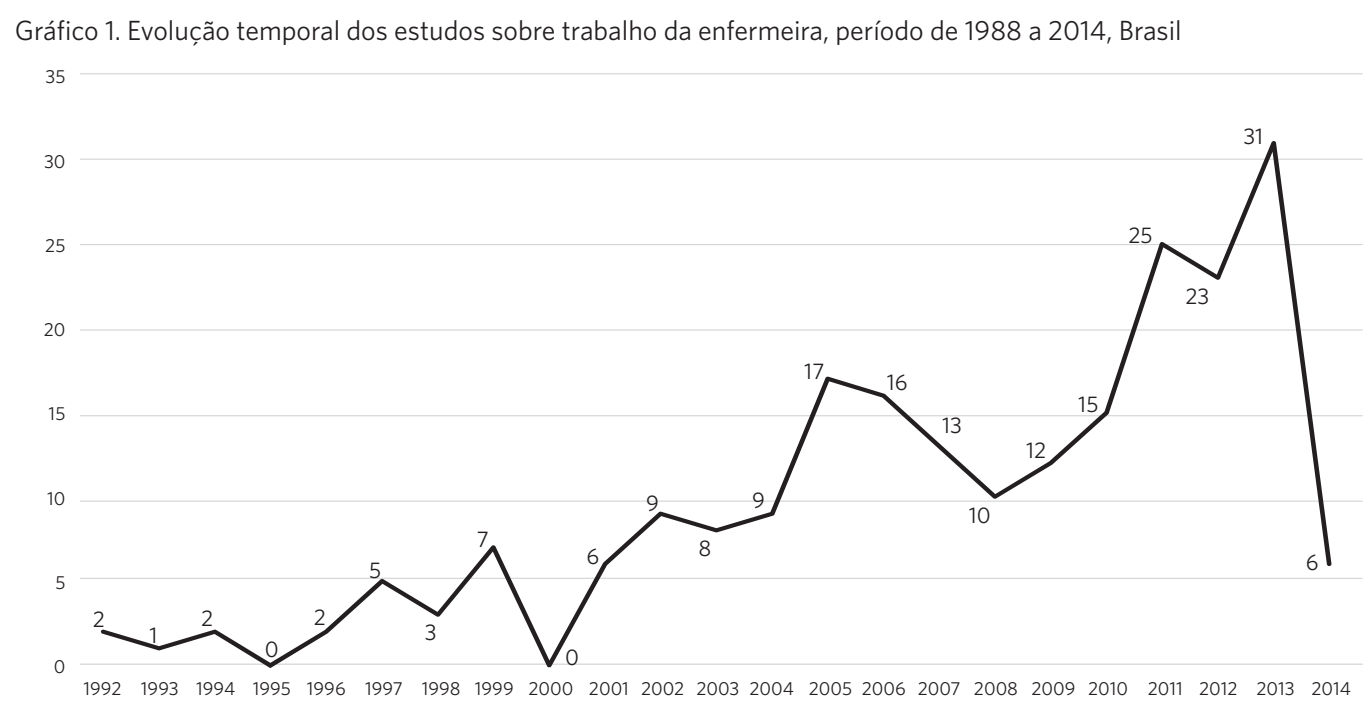

Fonte: Elaboração própria. SciELO, 2014. 
O crescimento do número de publicações sobre o tema pode indicar a ênfase que vem se dando, na área de Enfermagem, à formação pós-graduada, que contempla a realização e publicação de resultados de pesquisas, como atesta o aumento da titulação de mestres, desde o início da década de 1970, e de doutores, a partir do começo dos anos 1980, favorecendo o amadurecimento científico da área a partir de então (ROBAZZI, 2010; MANDÚ ET AL., 2011). De fato, da década de 1980 em diante, cresceu o número de programas de pós-graduação e de cursos, os quais provavelmente foram responsáveis pelo aumento em quantidade e qualidade da produção de conhecimentos científicos ou tecnológicos, bem como de periódicos científicos que possibilitaram maior divulgação dessa produção. A ascensão do número de estudos verificado entre os anos 2000 e 2010 também pode ser atribuída, por um lado, ao maior número de periódicos indexados na base de dados analisadas e, por outro, ao interesse pela produção de conhecimentos em relação ao tema trabalho da enfermeira, dado o desenvolvimento de políticas em saúde e o cenário de implantação do SUS, com a implementação de propostas de reorganização do modelo de atenção à saúde e ênfase nas ações preventivas, mas também pelos esforços na organização da atenção hospitalar e das urgências e emergências (BRASIL, 2006).
Desse modo, a curva ascendente de publicações verificada entre os anos 2011 e 2013 pode estar associada à emergência de questões concretas relacionadas com o trabalho da enfermeira nas duas décadas de implantação do SUS (PAIM, 2013) e também de expansão dos serviços vinculados ao Sams (SESTELO; BAHIA, 2014), implicando diferentes modos de pensar e organizar o trabalho da enfermeira, em articulação com as políticas e os sistemas de saúde vigentes.

\section{Distribuição dos estudos segundo o periódico em que foram publicados}

Com base na identificação do periódico no qual foi publicado cada resumo selecionado e distribuição dos estudos, a Revista LatinoAmericana de Enfermagem, publicada pela Universidade de São Paulo (USP), apresentou maior concentração de estudos relacionados com a temática, seguida da Revista Brasileira de Enfermagem, Revista Escola de Enfermagem USP; Texto e Contexto Enfermagem; Revista Escola Anna Nery; Acta Paulista Enfermagem; Ciência e Saúde Coletiva; Revista Gaúcha de Enfermagem e outros (tabela 1). Essa distribuição evidencia que os periódicos que se relacionam diretamente com a área de enfermagem concentram $93,24 \%$ de toda a produção analisada.

Tabela 1. Distribuição dos estudos sobre trabalho da enfermeira, segundo o periódico em que foram publicados, no período de 1988 a 2014, Brasil

\begin{tabular}{lrr}
\hline Periódico & Frequência & Percentual (\%) \\
\hline Revista Latino-Americana de Enfermagem & 49 & 22,07 \\
Revista Brasileira de Enfermagem & 48 & 21,63 \\
Revista Escola de Enfermagem da USP & 37 & 16,67 \\
Texto e Contexto Enfermagem & 29 & 13,06 \\
Revista Escola Ana Nery & 22 & 9,91 \\
Acta Paulista Enfermagem & 14 & 6,31 \\
Ciência e Saúde Coletiva & 09 & 4,05 \\
Revista Gaúcha de Enfermagem & 08 & 3,60 \\
Outros & 06 & 2,70 \\
\hline Total & $\mathbf{2 2 2}$ & $\mathbf{1 0 0}$ \\
\hline
\end{tabular}

Fonte: Elaboração própria. SciELO, 2014 


\section{Distribuição dos estudos segundo abordagem teórico-metodológica}

Em relação à abordagem teórico-metodológica, os estudos foram classificados a partir da indicação registrada no resumo dos artigos, a saber: a) resultados de pesquisas; b) relatos de experiências; c) estudos bibliográficos; d) estudos teóricos; e) outros. Os estudos que resultaram de pesquisas originais foram desagregados, distinguindo-se os que utilizam uma abordagem qualitativa, quantitativa e quantiqualitativa.

Entre os estudos selecionados (tabela 2), identifica-se predomínio de investigações que resultam de pesquisa, sendo a maioria qualitativas $(64,4 \%)$, e uma pequena parte quantitativas $(4,9 \%)$ e quantiqualitativas (2,2\%). Em segundo lugar, aparecem estudos teóricos, que correspondem a $13,5 \%$ da produção cientifica analisada, seguido dos estudos bibliográficos (9,5\%), relatos de experiências $(3,6 \%)$ e outros $(1,9 \%)$.

Tabela 2. Distribuição dos estudos sobre trabalho da enfermeira, segundo o tipo de abordagem metodológica no período de 1988 a 2014, Brasil

\begin{tabular}{lrr}
\hline Tipo de abordagem metodológica & N & \% \\
\hline A) Pesquisas & 143 & 64,4 \\
Estudos qualitativos & 11 & 4,9 \\
Estudos quantitativos & 5 & 2,2 \\
Estudos quantiqualitativos & $\mathbf{1 5 9}$ & $\mathbf{7 1 , 5}$ \\
\hline Subtotal & 30 & 13,5 \\
\hline B) Estudos teóricos & 21 & 9,5 \\
C) Estudos bibliográficos & 8 & 3,6 \\
D) Relatos de experiência & 4 & 1,9 \\
Outros & $\mathbf{2 2 2}$ & $\mathbf{1 0 0}$ \\
\hline Total & & \\
\hline
\end{tabular}

Fonte: Elaboração própria. SciELO, 2014.

Ao considerar que os estudos teóricos poderiam apontar de forma mais clara os diversos referenciais que vêm sendo utilizados pelos grupos de pesquisa que se dedicam ao tema 'trabalho da enfermeira', tais estudos foram lidos na íntegra, constatando-se que a maioria destes constituem ensaios que refletem a opinião das autoras sobre determinado tema, não constituindo de fato uma reflexão teórica sobre o trabalho da enfermeira. Os que apresentam conceitos e teorias podem ser classificados em dois grandes grupos: os estudos que se filiam a uma corrente 'estruturalista' e outros que se vinculam a uma perspectiva 'interacionista'. Entre os primeiros, identificou-se a referência à 'Teoria do Processo de Trabalho em Saúde'
(TAUBE; MEIER, 2007), à 'Teoria das Necessidades Humanas Básicas' (FURTADO; NÓBREGA, 2013), ao 'Materialismo Dialético' (PEREIRA; ALVES, 2004), à 'Teoria Institucionalista' (FRIEDRICH; SENA, 2002) e à 'Teoria da Intervenção Práxica da Enfermagem' (ERMEL; FRACOLLI, 2006). Todos esses enfoques admitem o cuidado como núcleo central do trabalho da enfermeira, a ser estruturado a partir das necessidades individuais e coletivas, sendo que os adeptos do 'materialismo dialético' introduzem, de forma mais contundente, a contradição entre o ideal e a realidade apresentada. Entre os segundos, percebe-se a utilização de teorias que se apoiam na 'psicodinâmica do trabalho' (FERREIRA ET AL., 2009), enfocando o sofrimento e a necessidade de reconhecimento 
social ou as relações de poder configuradas no cotidiano do trabalho, a 'arqueologia do saber' (ARAÚjo, 2007), a 'teoria do pensamento complexo' (PESTANA; ERDMANN; SOUSA, 2012) e a 'teoria do cuidado humano' (SANTOS ET AL., 2013). Há ainda os que analisam o trabalho da enfermeira a partir de aportes da 'teoria cultural' (PROCHNOW; LEITE; ERDMANN, 2005) Ou 'teoria do cotidiano' (OLIVEIRA; LOYOLA, 2006) e estudos baseados no 'interacionismo simbólico', notadamente na 'fenomenologia' (BRESSAN; SCATENA, 2002) ou na 'teoria das representações sociais' (REIS; ANDRADE, 2008).

\section{Distribuição dos estudos segundo área temática/mudanças no trabalho da enfermeira}

Os 222 estudos analisados abordam 5 temas/ áreas temáticas (gráfico 2): trabalhos da enfermeira em unidades de produção especificas ( $n=78)$; seguidos do trabalho da enfermeira a partir da introdução de novas tecnologias $(\mathrm{n}=72)$; características do sujeito/agente do trabalho $(n=46)$; relações interpessoais no trabalho da enfermeira $(n=19)$ e outros $(n=07)$.

Gráfico 2. Distribuição dos estudos sobre trabalho da enfermeira, segundo a área temática, período de 1988 a 2014, Brasil

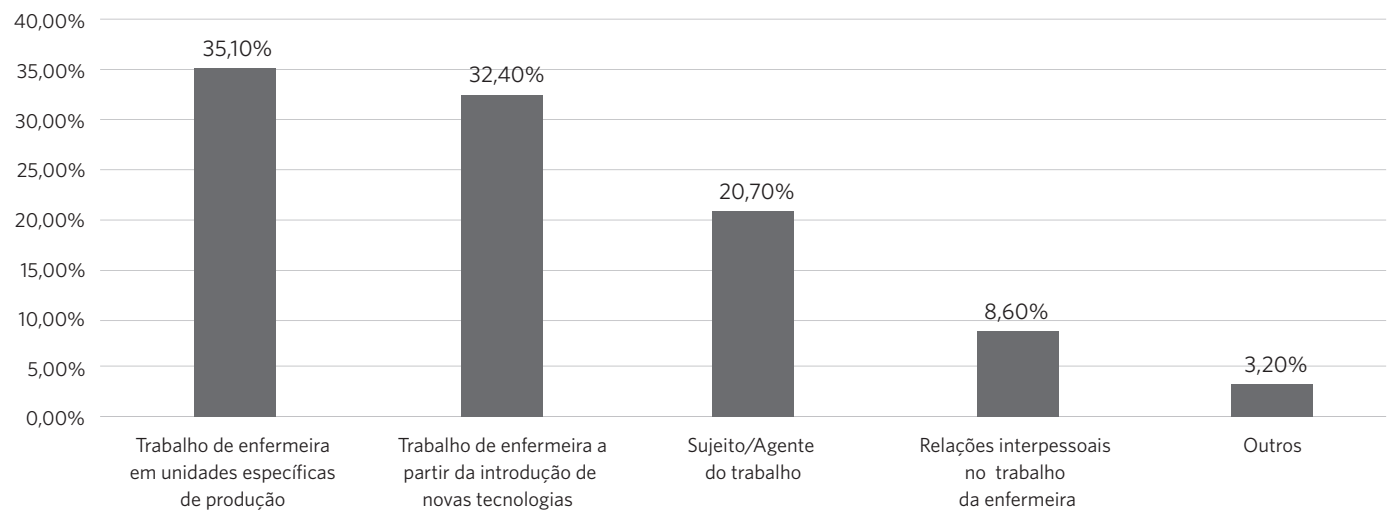

Fonte: Elaboração própria. SciELO, 2014

A maior parte dos estudos - 78 (35,1\%) - trata do trabalho da enfermeira(o) em unidades de produção específicas abordando as práticas, correlacionando-as com as especificidades das unidades produtivas. Desses, a proporção de estudos que analisam o trabalho da enfermeira no âmbito da atenção básica correspondem a $25 \%$ do total, enquanto a proporção de estudos que analisam trabalho da enfermeira, no âmbito de serviços de média e alta complexidade/densidade tecnológica, é bem mais representativa $(55,2 \%)$, o que evidencia que serviços especializados ainda constituem o cenário de práticas privilegiado. O SUS representa um grande mercado de trabalho para a enfermeira, com destaque para a sua inserção não apenas no Programa de Agentes Comunitários de Saúde (Pacs) e no Programa Saúde da Família (MACHADO, 2012), mas também, na gestão de sistemas, unidades e serviços de saúde (SOUZA, 2007).

Entre esses estudos, podem-se citar como exemplos, os que tratam das condições necessárias para execução do trabalho (COSTA; LIMA; ALMEIDA, 2003), competências e habilidades requeridas para o trabalho (PRADO; DIAS; CASTRO, 2014), organização/distribuição de tempo de trabalho em relação às atividades desenvolvidas (SOUZA; JERICÓ; PERROCA, 2013) e 
análise de custos das atividades (MARGARIDO; CASTILHO, 2006). Há também os que analisam ações especificas da enfermeira em face de determinado agravo ou situação de saúde (BEZERRA, 2011), incluindo os que destacam a participação da enfermeira na implantação e/ou execução de políticas/programas de saúde (PEREIRA; ALVES, 2004).

No que diz respeito à área temática organização do trabalho da enfermeira, com um total de 72 (32,4\%) estudos, observa-se a existência de duas perspectivas: a primeira se refere aos estudos que descrevem a introdução de tecnologias - materiais e imateriais (MERHY; FEUERWERKER, 2009), a exemplo de novos equipamentos (FONSECA; SANTOS, 2007), conhecimentos (FORTUNA ET AL., 2011), instrumentos (SILVA ET AL., 2011) - na prática da enfermeira; enquanto a segunda reporta-se aos estudos, em menor número, que abordam a redefinição do trabalho da enfermeira em função das mudanças da organização do sistema e nos serviços de saúde.

Na primeira perspectiva, chama a atenção o pequeno número de estudos que apontam a introdução da Sistematização da Assistência de Enfermagem (SAE) (ALVES ET AL., 2007; CORSO ET AL., 2013), que se constitui uma tecnologia própria da enfermagem, desenvolvida como método específico, visando articular conhecimentos e técnicas, ampliando a ação da enfermeira na resposta às necessidades individuais e coletivas.

Já na segunda, há os estudos que analisam diretrizes da Reforma Psiquiátrica (GONÇALVES; LANA, 2002), os estudos que discutem a introdução de protocolos assistenciais (MENEzes; D'INNOCENZO, 2013), incluindo a sistematização da assistência de enfermagem (ALVES ET AL., 2007), normas e rotinas hospitalares (BOCHI ET AL., 2007) e a organização do trabalho da enfermeira do ponto de vista das características da atenção básica (NASCIMENTO; NASCIMENTO, 2005) ou de mudanças organizacionais em hospitais (FONSECA; ALVES, 2002), e ainda a articulação entre os diversos níveis de atenção à saúde (BARATIERI; MANDU; MARCON, 2012) ou entre as dimensões assistenciais, gerenciais, educativas e de pesquisa do trabalho da enfermeira (HAUSMANN; PEDUZZI, 2009).

Dos 46 (20,7\%) estudos que tratam das características do sujeito/agente do trabalho, cerca de metade aborda a visão da enfermeira sobre o seu trabalho (SANCHES; CHRISTOVAM; SILVINO, 2006), e os demais focam na visão da enfermeira sobre aspectos específicos do trabalho, como prazer (BATISTA ET AL., 2005) e sofrimento (CAMPOS; DAVID; SOUZA, 2014), grau de autonomia (VARGAS; RAMOS, 2010) e atributos necessários para essa escolha profissional (ROSCANI; GUIRARDELLO, 2010).

Dos 19 (8,6\%) estudos que tratam das relações interpessoais no trabalho, a maioria enfoca as relações entre enfermeira e usuários (PROCHET ET AL., 2012). Aparecem também estudos sobre as relações entre a enfermeira e os demais profissionais de saúde (PROCHNOW ET AL., 2007) e as relações institucionais entre enfermeiras e o ente empregador (ROSSI, 1994), bem como estudos que apontam a violência institucional (BARBOSA ET AL., 2011) e o assédio moral (CAHÚ ET AL., 2014), o que aponta a provável existência de um processo de deterioração das relações interpessoais no ambiente de trabalho (FRANCO; DRUCK; SELIGMANN-SILVA, 2010) assim como a fragilização do reconhecimento social e da valorização simbólica da enfermeira, relacionadas, inclusive, com questões de gênero.

\section{Conclusões}

A caracterização da produção científica nacional registrada na base SciELO sobre o trabalho da enfermeira, no período de 1988 a 2014, evidencia, em primeiro lugar, o aumento do número de publicações na década de 2000, provavelmente associada ao crescente número de enfermeiras que se inseriram em cursos de pós-graduação com interesse em analisar questões relacionadas com o trabalho profissional, ainda que enfermeiras não vinculadas a tais cursos possam 
também estar se interessando em escrever e publicar trabalhos nessa linha.

A distribuição dos estudos segundo revistas científicas da área de saúde, por sua vez, revela uma concentração em revistas especializadas na área de enfermagem, fato que possivelmente reflete certo insulamento, circunscrevendo as discussões à enfermagem, ainda que, entre as categorias profissionais que compõem as equipes de saúde, a enfermagem represente o maior contingente (MACHADO, 2015).

A identificação das abordagens metodológicas utilizadas nos trabalhos selecionados aponta uma ênfase em estudos de natureza qualitativa, derivada de pesquisas empíricas sobre o trabalho/processo de trabalho (TAUBE; MEIER, 2007; GONÇALVES; LANA, 2002; SANCHES; CHRISTOVAM; SILVINO, 2006), a prática (ARAÚJO, 2007; SOUZA; JERICÓ; PERROCA, 2013; ROSSI, 1994) OU O cuidado (PESTANA; ERDMANN; SOUSA, 2012; PROCHNOW; LEITE; ERDMANN, 2005; FORTUNA ET AL., 2011) da enfermeira, embora existam trabalhos teóricos que evidenciam a multiplicidade de teorias e conceitos provindas das ciências humanas e sociais incorporadas às investigações acerca do trabalho da enfermeira.

O mapeamento dos temas abordados revelou uma preponderância de estudos na categoria 'trabalho da enfermeira em unidades de produção especificas', o que evidencia uma preocupação das pesquisadoras em relatar pequenas mudanças na pratica profissional, sem necessariamente problematizar a reorganização do trabalho da enfermeira em função das propostas de reorientação do modelo de atenção à saúde que vêm sendo discutidas e implementadas no âmbito do SUS (TEIXEIRA; VILLASBÔAS, 2014).

As mudanças apontadas nesses estudos referem-se, majoritariamente, à introdução de novas tecnologias e novos modos de organização do trabalho da enfermeira, em conformidade com as mudanças técnico-científicas da área, inegavelmente ocorridas ao longo das últimas décadas. Além disso, apontam outras mudanças ocorridas no trabalho da enfermeira, a exemplo da deterioração das condições de trabalho, o sofrimento no trabalho, a perda da autonomia, a deterioração das relações interpessoais e a sobrecarga de funções e tarefas.

Apesar dos esforços empreendidos no estudo desses processos, tais mudanças ainda são analisadas isoladamente, em sua maioria, vistas de forma desarticulada, na medida em que focam aspectos parciais do processo de trabalho (conhecimentos, tecnologias, objetos etc.), sem discutir as consequências de mudanças em cada um desses elementos sobre as dimensões assistenciais, gerenciais, educativas e de pesquisa do trabalho da enfermeira, nem tampouco os determinantes econômicos, políticos e sociais que as impulsionam.

Isso pode estar refletindo, por um lado, a incorporação acelerada de novos conhecimentos e tecnologias no trabalho em saúde, que acompanha o avanço científico e determina o surgimento de especialidades e subespecialidades que se apropriam de parcelas do trabalho na área. Estudos sobre tais processos contribuem para o conhecimento do que vem ocorrendo no âmbito do trabalho da enfermeira, principalmente uma tendência à tecnificação do cuidado, reduzido à execução de procedimentos (MERHY; FEUERWERKER, 2009), processo que circunscreve o trabalho da enfermeira a mera execução de tarefas. Por outro lado, alguns dos trabalhos analisados apontam que o uso de tecnologias desenvolvidas pela enfermagem e para a enfermagem pode promover espaços criativos no cotidiano do trabalho e ressignificação das práticas (ALVES ET AL., 2007; CORSO ET AL., 2013; ÉVORA; DALRI, 2002).

Outro aspecto a ser destacado desses estudos é a compreensão de que o progresso científico e tecnológico, ao tempo que tem exigido a incorporação de novos elementos pessoais e atitudinais ao trabalho da enfermeira, sejam esses representados pela competência técnica ou pelas habilidades necessárias as ações (PRADO; DIAS; CASTRO, 2014), 
são contraditórios ao exercício da autonomia, limitando-a (KRAEMER; DUARTE; KAISER, 2011; VARGAS; RAMOS, 2010). Há uma redução das possibilidades de governo de si da enfermeira nos atuais cenários do trabalho (SILVA; CRUZ, 2008).

Também merece discussão, com base nos estudos que abordam a organização do trabalho da enfermeira, o fato das mudanças epidemiológicas e sociais que vêm ocorrendo no Brasil nas últimas décadas estar gerando novas necessidades de saúde (NASCIMENTO; NASCIMENTO, 2005; BARATIERI; MANDU; MARCON, 2012), as quais induzem mudanças no objeto de trabalho da enfermeira, que se amplia, conforme indicam os estudos, agregando a família e as comunidades. Entretanto, apesar dessa ampliação do objeto, vale a pena ressaltar que as necessidades de saúde são parte da complexa dinâmica social brasileira, expressando interesses diversos, os quais limitam ou potencializam a ação da enfermeira.

Nessa perspectiva, as propostas de reversão do modelo de atenção médico-assistencial e hospitalocêntrico (TEIXEIRA; VILLASBÔAS, 2014; PAIM, 2012) têm produzido o deslocamento do foco nos aspectos individuais e biológicos, valorizando ações de prevenção e promoção à saúde, individuais e coletivas, o que se reflete nos estudos sobre o trabalho da enfermeira, com ênfase em sua inserção na atenção básica (PEREIRA; ALVES, 2004; ERMEL; FRACOLLI, 2006; FORTUNA ET AL., 2011) e em outros âmbitos, a exemplo da atenção obstétrica (SILVA ET AL., 2011; ALVES ET AL., 2007) e pediátrica/hospitalar (SANTOS ET AL., 2013), com a introdução, inclusive, de práticas integrativas que privilegiam aspectos relacionais. Apesar de estas serem experiências pontuais, há indícios de abertura de espaços de criação no trabalho da enfermeira que permitem a inserção de novos elementos para a compreensão das variadas situações e das formas de enfrentá-las.

Todavia, os trabalhos analisados apontam também que a mudança no modo de organização do trabalho da enfermeira induzida pelos novos modelos de gestão adotados pelas instituições, notadamente as hospitalares, com vistas e ao aumento da eficiência, tem promovido certo acréscimo das atividades, traduzido na sobrecarga de trabalho, com ênfase nas ações burocráticas (SANCHES; CHRISTOVAM; SILVINO, 2006) e perda de autonomia (VARGAS; RAMOS, 2010). As propostas de gestão, que vêm sendo desenvolvidas e implementadas, portanto, resultam, na maioria das vezes, em 'modernizações conservadoras', desestimulando o desenvolvimento dos profissionais, gestores e usuários (FONSECA; ALVES, 2002; GALLO, 2009).

Configura-se, portanto, uma contradição entre o desenvolvimento das potencialidades da enfermeira e as características da lógica produtiva vigente que prima pela intensificação do controle e estimulo à competitividade, o que acarreta, inclusive, medo da demissão (FRANCO; DRUCK; SELIGMANN-SILVA, 2010) e sofrimento no trabalho (FEREIRA ET AL., 2009). Alguns estudos já relacionam o sofrimento às condições ambientais e infraestruturais das instituições de saúde (COSTA; LIMA; ALMEIDA, 2003), cuja deterioração resulta das escolhas e condução dos distintos projetos políticos institucionais, apontando, ainda, a falta de reconhecimento do trabalho da enfermeira (FONSECA; ALVES, 2002). Condições de trabalho se referem às especificidades de quem o realiza e envolve, desde instrumentos adequados em quantidade e qualidade até os conhecimentos para operá-los, o ambiente, espaço físico para a realização do trabalho (PIRES; LORENZETTI; GELBCKE, 2010) e também as relações contratuais.

No que diz respeito à deterioração das relações interpessoais, alguns trabalhos apontam a intensificação da competitividade nas relações entre a enfermeira e os demais profissionais ou trabalhadores da saúde (BATISTA ET AL., 2005), mas também nas relações entre a enfermeira e a equipe de enfermagem (URBANETTO; CAPELLA, 2004), o que, segundo os autores, enfraquece as possibilidades de conformação de trabalhos cooperativos e mobilização para lutas conjuntas. Por outro lado, cabe ressaltar que a existência de 
relações contratuais precárias não foi referida nos estudos analisados. Do mesmo modo, chama a atenção o fato de que não foram encontrados trabalhos que analisem a atuação política das enfermeiras, por meio de suas entidades representativas, como protagonistas das mudanças que vêm ocorrendo em sua prática profissional.

Apesar de o estudo limitar-se à análise da produção cientifica nacional e centrar-se em um período de transformações políticas e organizacionais do setor da saúde no Brasil, cabe registrar que os achados sobre as mudanças no trabalho da enfermeira nos estudos analisados corroboram alguns estudos internacionais, notadamente no que se refere aos novos modos de organização do trabalho da enfermeira, seja em conformidade com o avanço cientifico e tecnológico e/ou com as novas engenharias organizacionais, com destaque para o decréscimo das condições de trabalho, o sofrimento no trabalho, a deterioração das relações interpessoais e a sobrecarga de funções e tarefas, tendo como consequência o abandono da profissão (DUVALL; ANDREWS, 2010; TOH; ANG; DEVI, 2012).
Para concluir, é importante salientar que apesar da grande contribuição da produção científica nacional analisada ao conhecimento acerca das características do trabalho da enfermeira no Brasil contemporâneo, em seus múltiplos aspectos tecnológicos, organizacionais e relacionais, $\mathrm{o}$ tema 'trabalho da enfermeira', por sua complexidade e importância para o campo da saúde coletiva, exige analises que agreguem a correlação dessas mudanças com os processos políticos e a reorganização dos sistemas de saúde, que atravessam o cenário atual de construção do SUS e de expansão do Sams, permitindo compreender o presente e vislumbrar o futuro.

\section{Colaboradores}

Ednir Assis Souza desenvolveu a pesquisa, a análise e interpretação dos dados e a elaboração do manuscrito. Carmen Fontes Teixeira orientou a pesquisa, a concepção do manuscrito, realizou a revisão crítica e a aprovação da versão final do manuscrito. Mariluce Karla Bomfim de Souza participou da revisão crítica do manuscrito. 


\section{Referências}

\author{
ALMEIDA, M. C. P.; ROCHA, S. M. M. O Trabalho de \\ enfermagem. São Paulo: Cortez, 1997.
}

ALVES, A. R. et al. Aplicação do Processo de Enfermagem: estudo de caso com uma puérpera. Revista Brasileira de Enfermagem, Brasília, DF, v. 60, n. 3, p. 344-47, maio/jun., 2007. Disponível em: <http://www.scielo.br/scielo.php?pid=S0034$-71672007000300019 \&$ script $=$ sci_abstract\&tlng=pt $>$. Acesso em: 15 maio 2016.

ARAÚJO, L. F. S. Processos de subjetivação inscritos na constituição da experiência de si da(o) enfermeira(o) nas práticas assistenciais de um cenário de trabalho exemplar: a Unidade de Terapia Intensiva. Texto Contexto Enfermagem, Florianópolis, v. 16, n. 1, p. 180-1, jan./mar., 2007.

\section{BARATIERI, T.; MANDU, E. N. T.; MARCON, S. S.} Longitudinalidade no trabalho do enfermeiro: relatos da experiência profissional. Revista da Escola de Enfermagem da USP, São Paulo, v. 46, n. 5, p. 1260-1267, out. 2012. Disponível em: <http://www.scielo.br/scielo. php?script=sci_arttext\&pid=S0080-62342012000500031 $>$. Acesso em: 15 maio 2016.

BARBOSA, R. B. et al. Violência psicológica na prática profissional da enfermeira. Revista da Escola de Enfermagem da USP, São Paulo, v. 45, n. 1, p. 26-32, mar. 2011. Disponível em: <http://www.scielo.br/pdf/ reeusp/v45n1/04.pdf>. Acesso em: 16 maio 2016.

BATISTA, A. A. V. et al. Fatores de motivação e insatisfação no trabalho do enfermeiro. Revista Escola de Enfermagem da USP, São Paulo, v. 39, n. 1, p. 85-91, mar. 2005. Disponível em: <http://www.scielo.br/pdf/ reeusp/v39nl/allv39nl.pdf>. Acesso em: 16 maio 2016.

BEZERRA, P. M. Processos de trabalho do enfermeiro durante surtos de raiva humana no Estado do Pará, Brasil. Revista Brasileira de Enfermagem, Brasília, DF, v. 64, n. 1, p. 78-83, jan./fev. 2011. Disponível em: <http:// www.scielo.br/scielo.php?script=sci_arttext\&pid =S0034-71672011000100012>. Acesso em: 16 maio 2016.
BRASIL. Ministério da Saúde. Política Nacional de Atenção às Urgências. 3. ed. Brasília, DF: Ministério da Saúde, 2006.

BRESSAN, V. R.; SCATENA, C. M. O cuidar do doente mental crônico na perspectiva do enfermeiro: um enfoque fenomenológico. Revista Latino-Americana de Enfermagem, Ribeirão Preto, v. 10, n. 5, p. 682-689, set./ out. 2002. Disponível em: <http://www.scielo.br/scielo. php?pid=S0104-11692002000500009\&script=sci_abstract>. Acesso em: 16 maio 2016.

BOCHI, S. C. M. et al. Familiares visitantes e acompanhantes de adultos e idosos hospitalizados: análise da experiência na perspectiva do processo de trabalho em enfermagem. Revista Latino-Americana de Enfermagem, Ribeirão Preto, v. 15, n. 2, p. 304-310, mar./abr. 2007. Disponível em: <http://www.scielo.br/ scielo.php?pid=S0104-11692007000200017\&script=sci abstract\&tlng=pt>. Acesso em: 16 maio 2016.

CAHÚ, G. R. P. et al. Situações de assédio moral vivenciadas por enfermeiros no ambiente de trabalho. Acta Paulista de Enfermagem, São Paulo, v. 27, n. 2, p. 151-156, mar./abr. 2014. Disponível em: <http://www.scielo.br/ scielo.php?pid=S0103-21002014000200011\&script=sci abstract\&tlng=es>. Acesso em: 16 maio 2016.

CAMPOS, J. F.; DAVID, H. M. S. L; SOUZA, N. V. D. O. Prazer e sofrimento: avaliação de enfermeiros intensivistas à luz da psicodinâmica do trabalho. Escola Anna Nery, Rio de Janeiro, v. 18, n. 1, p. 90-95, jan./mar. 2014. Disponível em: <http:// www.scielo.br/scielo.php?script=sci_arttext\&pid $=$ S1414-81452014000100090>. Acesso em: 16 maio 2016.

CORSO, N. A. A. et al. Sistematização da Assistência de Enfermagem para acompanhamento ambulatorial de pacientes com esclerose múltipla. Revista Escola de Enfermagem da USP, São Paulo, v. 47, n. 3, p. 750-755, jun. 2013. Disponível em: <http:// www.scielo.br/scielo.php?script=sci_arttext\&pid $=$ S0080-62342013000300750>. Acesso em: 16 maio 2016. 
COSTA, J. R. A.; LIMA, J. V.; ALMEIDA, P. C. Stress no trabalho do enfermeiro. Revista Escola de Enfermagem da USP, São Paulo, v. 37, n. 3, p. 63-71, set. 2003.

Disponível em: <http://www.ee.usp.br/reeusp/upload/ pdf/170.pdf>. Acesso em: 16 maio 2016.

DONNANGELO, M. C. F. Medicina e sociedade: o médico e seu mercado de trabalho. São Paulo: Pioneira, 1975.

DUVALL, J. J.; ANDREWS, D. R. Using a structured review of the literature to identify key factors associated with the current nursing shortage. Journal of Profissional Nursing, Philadelphia, v. 26, n. 5, p. 309-17, set./out. 2010. Disponível em: < https://www.ncbi.nlm. nih.gov/pubmed/20869031>. Acesso em: 16 maio 2016.

ERMEL, R. C.; FRACOLLI, L. A. O trabalho das enfermeiras no Programa de Saúde da Família em Marília/SP. Revista Escola de Enfermagem da USP, São Paulo, v. 40, n. 4, p. 533-9, dez. 2006. Disponível em: <http://www.scielo.br/scielo.php?pid=S0080$-62342006000400012 \&$ script $=$ sci_abstract\&tlng=pt $>$. Acesso em: 16 maio 2016

ÉVORA, Y. D. M.; DALRI, M. C. B. O uso do computador como ferramenta para a implantação do processo de enfermagem. Revista Brasileira de Enfermagem, Brasília, DF, v. 24, n. 6, p. 709-713, nov./dez. 2002. Disponível em: <http://www.scielo.br/pdf/reben/ v55n6/v55n6a15.pdf>. Acesso em: 16 maio 2016.

FEREIRA, E. M. et al. Prazer e sofrimento no processo de trabalho do enfermeiro docente. Revista Escola de Enfermagem da USP, São Paulo, v. 43, n. 2, p. 1292-1296, dez. 2009. Disponível em: <http://www.scielo.br/pdf/ reeusp/v43nspe2/a25v43s2.pdf >. Acesso em: 16 maio 2016.

FONSECA, C. M. B. M.; SANTOS, M. L. Tecnologias da informação e cuidado hospitalar: reflexões sobre o sentido do trabalho. Ciência Et Saúde Coletiva, Rio de Janeiro, v. 12, n. 3, p. 699-708, maio/jun. 2007. Disponível em: <http://www. scielo.br/scielo.php?script=sci_arttext\&pid $=$ S1413-81232007000300020>. Acesso em: 15 maio 2016.
FONSECA, M. G.; ALVES, M. Trabalho do enfermeiro em um contexto de mudanças organizacionais. Revista Brasileira de Enfermagem, Brasília, DF, v. 55, n. 5, p. 600-604, set./out. 2002. Disponível em: <http:// www.scielo.br/scielo.php?script=sci_arttext\&pid $=$ S0034-71672002000500020 >. Acesso em: 15 maio 2016.

FORTUNA, C. M. F. et al. O enfermeiro e as práticas de cuidados coletivos na estratégia saúde da família. Revista Latino-Americana de Enfermagem, Ribeirão Preto, v. 19, n. 3, maio/jun. 2011. Disponível em: <http:// www.scielo.br/pdf/rlae/v19n3/pt_18.pdf $>$. Acesso em: 30 maio 2014.

FRANCO, T.; DRUCK, G.; SELIGMANN-SILVA, E. As novas relações de trabalho, o desgaste mental do trabalhador e os transtornos mentais no trabalho precarizado. Revista Brasileira de Saúde Ocupacional, São Paulo, v. 35, n. 122, p. 229-248, 2010. Disponível em: $<$ http://www.scielo.br/scielo.php?script=sci_arttext\& pid $=$ S0303-76572010000200006 $>$. Acesso em: 15 maio 2016.

FRIEDRICH, D. B.; SENA, R. R. Um novo olhar sobre o cuidado no trabalho da enfermeira em unidades básicas de saúde em Juiz de Fora- MG. Revista Latino-Americana de Enfermagem, Ribeirão Preto, v. 10, n. 6, p. 772-779, nov./dez. 2002. Disponível em: <http://www.scielo.br/scielo.php?pid=S0104$-11692002000600004 \&$ script=sci_abstract $\&$ tlng=eses $>$. Acesso em: 30 maio 2014.

FURTADO, L. G.; NÓBREGA, M. M. L. Modelo de atenção crônica: inserção de uma teoria de enfermagem. Texto Contexto Enfermagem, Florianópolis, v. 22, n. 4, out./dez. 2013. Disponível em: <http:// www.scielo.br/scielo.php?script=sci_arttext\&pid =S0104-07072013000400039>. Acesso em: 30 maio 2015 .

GALLO, E. Alienação, inovação e cotidiano organizacional. In: MANDARINO, A. C. S.; GOMBERG, E. (Org.). Leitura de novas tecnologias e saúde. Salvador: Edufba, 2009. p. 55-72.

GONÇALVES, A. M. C.; LANA, F. C. F. Reforma 
psiquiátrica e sua articulação com o processo de trabalho do enfermeiro. Revista Brasileira de Enfermagem, Brasília, DF, v. 55, n. 5, p. 600-604, set./out. 2002. Disponível em: <http://www. scielo.br/scielo.php?script=sci_arttext\&pid $=$ S0034-71672002000500019>. Acesso em: 30 maio 2015

HAUSMANN, M.; PEDUZZI, M. Articulação entre as dimensões gerencial e assistencial do processo de trabalho do enfermeiro. Texto Contexto Enfermagem, Florianópolis, v. 18, n. 2, p. 258-265, abr./jun. 2009. Disponível em: <http://www.scielo.br/pdf/tce/ v18n2/08.pdf>. Acesso em: 15 maio 2016.

KRAEMER, F. Z.; DUARTE, M. L. C.; KAISER, D. E. Autonomia e trabalho do enfermeiro. Revista Gaúcha de Enfermagem, Porto Alegre, v. 32, n. 3, p. 487-494, set. 2011. Disponível em: <http:// www.scielo.br/scielo.php?script=sci_arttext\&pid $=$ S1983-14472011000300008 $>$. Acesso em: 20 maio 2016.

MACHADO, M. H. Relatório sobre o Perfil da Enfermagem no Brasil. 2015. Disponível em: <http:// portal.fiocruz.br/pt-br/content/pesquisa-inedita-traca-perfil-da-enfermagem-no-brasil>. Acesso em: 20 jun. 2015.

MACHADO, M. H. Trabalho e emprego em saúde. In: GIOVANELLA, L. et al (Org.). Políticas e sistemas de saúde no Brasil. 2. ed. Rio de Janeiro: Fiocruz, 2012, p. 259-278.

MANDÚ, E. N. T. et al. Literatura brasileira sobre o trabalho de enfermagem fundamentada em categorias marxianas. Revista Brasileira de Enfermagem, Brasília, DF, v. 64, n. 4, p. 766-773, jul./ago. 2011. Disponível em: <http://www.scielo.br/scielo.php?script=sci_arttext\& pid=S0034-71672011000400021 $>$. Acesso em: 20 jun. 2015.

MARGARIDO, E. S.; CASTILHO, V. Aferição do tempo e do custo médio do trabalho da enfermeira na consulta de enfermagem. Revista da Escola de Enfermagem da USP, Ribeirão Preto, v. 40, n. 3, p. 427-433, set. 2006. Disponível em: <http:// www.scielo.br/scielo.php?script=sci_arttext\&pid $=$ S0080-62342006000300016 $>$. Acesso em: 15 maio 2016.

MELO, C. M. M. Divisão social do trabalho e enfermagem. São Paulo: Cortez, 1986.

MENDES-GONÇALVES, R. B. Tecnologia e organização social das práticas de saúde: características tecnológicas do processo de trabalho na rede estadual de centros de saúde de São Paulo. São Paulo: Hucitec, 1994.

MENEZES, P. I. B.; D’INNOCENZO, M.

Dificuldades vivenciadas pelo enfermeiro na utilização de indicadores de processos. Revista Brasileira de Enfermagem, Brasília, DF, v. 66, n. 4, p. 571-7, jul./ago. 2013. Disponível em: <http:// www.scielo.br/scielo.php?script=sci_arttext\&pid =S0034-71672013000400016>. Acesso em: 15 maio 2016.

MERHY, E.; FEUERWERKER, L. C. Novo olhar sobre as tecnologias de saúde: uma necessidade contemporânea. In: MANDARINO, A. C. S; GOMBERG, E. (Org.). Leitura de novas tecnologias e saúde. Salvador: EDUFBA, 2009, p.29-54.

NASCIMENTO, M. S.; NASCIMENTO, M. A. A. Prática da enfermeira no Programa de Saúde da Família: a interface da vigilância da saúde versus as ações programáticas em saúde. Ciência \&t Saúde Coletiva, Rio de Janeiro, v. 10, n. 2, p. 333-345, abr./jun. 2005. Disponível em: $<$ http://www.scielo.br/scielo.php?script=sci_artt ext\&pid=S1413-81232005000200011 $>$. Acesso em: 15 maio 2016.

OLIVEIRA, R. M. P.; LOYOLA, C. M. D. Pintando novos caminhos: a visita domiciliar em saúde mental como dispositivo de cuidado em enfermagem. Escola Anna Nery, Rio de Janeiro, v. 10, n. 4, p. 645-51, dez. 2006. Disponível em: <http://www.scielo.br/scielo. php?pid=S1414-81452006000400005\&script=sci_abstract>. Acesso em: 15 maio 2016.

PACKER, A. L. et al. (Org.). SciELO 15 Anos de Acesso Aberto: um estudo analítico sobre Acesso Aberto e comunicação científica. Paris: Unesco, 2014. 
PAIM, J. S. Reforma Sanitária Brasileira: contribuição para compreensão e crítica. Salvador: EDUFBA; Rio de Janeiro: Fiocruz, 2008.

PAIM, J. S. et al. O sistema de saúde brasileiro: história, avanços e desafios. Série Saúde no Brasil, Rio de Janeiro, n. 1, p. 11-31, maio 2011.

PAIM, J. S. Modelos de atenção à saúde no Brasil. In: GIOVANELLA, L. et al. (Org.). Políticas e sistemas de saúde no Brasil. 2. ed. Rio de Janeiro: Fiocruz, 2012, p. 459-492

A Constituição Cidadã e os 25 anos do Sistema Único de Saúde (SUS). Cadernos Saúde Pública, Rio de Janeiro, v. 29, n. 10, p. 1927-1953, out. 2013. Disponível em: <http://www.scielo.br/scielo.php?script=sci_ arttext\&pid=S0102-311X2013001000003 $>$. Acesso em: 24 maio 2016.

PEREIRA, C. O. M.; ALVES, M. A participação do enfermeiro na implantação do Programa de Saúde da Família em Belo Horizonte. Revista Brasileira de Enfermagem, Brasília, DF, v. 57, n. 3, p. 311-315, jun. 2004. Disponível em: <http://www.scielo.br/scielo. php?pid=S0034-71672004000300011\&script=sci abstract\&tlng=pt $>$. Acesso em: 24 maio 2016.

PESTANA, A. L.; ERDMANN, A. L.; SOUSA, F. G. M. Emergindo a complexidade do cuidado de enfermagem ao ser em morte encefálica. Escola Anna Nery, Rio de Janeiro, v. 16, n. 4, p. 734-740, out./dez. 2012. Disponível em: $<$ http://www.scielo.br/scielo.php?script=sci_artt ext\&pid=S1414-81452012000400013 $>$. Acesso em: 24 maio 2016

PINTO, I. C. et al. Trabalho e educação em saúde no Brasil: tendências da produção científica entre 19902010. Ciência \&t Saúde Coletiva, Rio de Janeiro, v. 18, n. 6, p. 1525-34, jun. 2013. Disponível em: <http://www. scielo.br/pdf/csc/v18n6/02.pdf >. Acesso em: 24 maio 2016.

PIRES, D. Hegemonia Médica na Saúde e na Enfermagem. São Paulo: Cortez, 1989.

PIRES D. E. P.; LORENZETTI, J.; GELBCKE, F. L.
Enfermagem: condições de trabalho para um fazer responsável. In: CONGRESSO BRASILEIRO DE ENFERMAGEM, 62., 2010. Anais... Florianópolis: CBEN, 2010.

PRADO, R. T.; DIAS, S. M.; CASTRO, E. A. B. Competências e habilidades para atuação do enfermeiro em bancos de olhos. Texto Contexto Enfermagem, Florianópolis, v. 23, n. 1, p. 47-55, jan./mar. 2014. Disponível em: <http://www.scielo.br/pdf/tce/v23nl/ pt_0104-0707-tce-23-01-00047.pdf >. Acesso em: 24 maio 2016

PROCHET, T. C. et al. Afetividade no processo de cuidar do idoso na compreensão da enfermeira. Revista da Escola de Enfermagem da USP, Ribeirão Preto, v. 46, n. 1, p. 96-102, fev. 2012. Disponível em: <http:// www.scielo.br/scielo.php?script=sci_arttext\&pid $=$ S0080-62342012000100013 >. Acesso em: 12 maio 2016.

PROCHNOW, A. G.; LEITE, J. L.; ERDMANN, A. L. Teoria interpretativa de Geertz e a gerência do cuidado: visualizando a prática social do enfermeiro. Revista Latino-Americana de Enfermagem, Ribeirão Preto, v. 13, n. 4, p. 583-590, jul./ago. 2005. Disponível em: $<$ http://www.scielo.br/scielo.php?script=sci_arttext\& pid=S0104-11692005000400018 >. Acesso em: 14 maio 2016.

PROCHNOW, A. G. et al. O conflito como realidade e desafio cultural no exercício da gerência do enfermeiro. Revista da Escola de Enfermagem da USP, Ribeirão Preto, v. 41, n. 4, p. 542-50, dez. 2007. Disponível em: $<$ http://www.scielo.br/scielo.php?script=sci_arttext\&p id=S0080-62342007000400002 $>$. Acesso em: 15 maio 2016.

REIS, C. B.; ANDRADE, S. M. O. Representações sociais das enfermeiras sobre a integralidade na assistência à saúde da mulher na rede básica. Ciência \& Saúde Coletiva, Rio de Janeiro, v. 13, n. 1, p.61-70, jan./fev. 2008. Disponível em: <http://www.scielo.br/scielo. php?pid=S1413-81232008000100011\&script $=$ sci_ abstract\&tlng=pt>. Acesso em: 15 maio 2016.

ROBAZZI, M. L. C. C. O desempenho da pós-graduação 
stricto sensu em Enfermagem e a busca pela excelência. Revista Latino-Americana de Enfermagem, Ribeirão Preto, v. 18, n. 5, set./out. 2010. Disponível em: <http:// www.scielo.br/pdf/rlae/v18n5/pt_01.pdf >. Acesso em: 12 jul. 2015.

ROSCANI, A. N. C. P.; GUIRARDELLO, E. B. Demandas de atenção no ambiente de trabalho e capacidade de direcionar atenção do enfermeiro. Revista Latino-Americana de Enfermagem, Ribeirão Preto, v. 18, n. 4, jul./ago. 2010. Disponível em: <htpp://www.scielo. br/pdf/rlae/v18n14/pt_17.pdf >. Acesso em: 15 jun.2014.

ROSSI, L. A. A prática de enfermagem em uma Unidade de Queimados: análise dos discursos dos enfermeiros. Revista Brasileira de Enfermagem, Brasília, DF, v. 47, n. 2, p. 100-107, abr./jun. 1994. Disponível em: $<$ http://www.scielo.br/scielo.php?script=sci_arttext\& pid=S0034-71671994000200003 $>$. Acesso em: 15 jun. 2016.

SANCHES, V. F.; CHRISTOVAM, B. P.; SILVINO, Z. R. Processo de trabalho do gerente de enfermagem em unidade hospitalar: uma visão dos enfermeiros. Escola Anna Nery, Rio de Janeiro, v. 10 n. 2, p. 214-20, ago. 2006. Disponível em: <http:// www.scielo.br/scielo.php?script=sci_arttext\&pid $=$ S1414-81452006000200007>. Acesso em: 15 maio 2016.

SANTOS, M. R. et al. Desvelando o cuidado humanizado: percepções de enfermeiros em oncologia pediátrica. Texto Contexto Enfermagem, Florianópolis, v. 22, n. 3, p. 646-53, jul./set. 2013. Disponível em: <http:// www.scielo.br/scielo.php?script=sci_arttext\&pid $=$ S0104-07072013000300010 $>$. Acesso em: 15 maio 2016.

SESTELO, J.; BAHIA, L. Sistemas de Atenção Médica Suplementar (SAMS): breve histórico e modalidades desenvolvidas no Brasil (seguro-saúde, medicina de grupo, cooperativas médicas, autogestão e outras). In: PAIM, J. S.; ALMEIDA FILHO, N. Saúde Coletiva: teoria e prática. Rio de Janeiro: Medbook, 2014, p. 139-50.

SILVA, I. A. S.; CRUZ, E. A. O Trabalho da enfermeira intensivista. Revista Escola de Enfermagem da USP, São
Paulo, v. 42, n. 3, p.546-53, set. 2008. Disponível em: $<$ http://www.scielo.br/pdf/reeusp/v42n3/v42n3a19. pdf $>$. Acesso em: 15 maio 2016.

SILVA, L. M. et al. Uso da bola suíça no trabalho de parto. Acta Paulista de Enfermagem, São Paulo, v. 24, n. 5, p. 656-662, 2011. Disponível em: <http:// www.scielo.br/scielo.php?script=sci_arttext\&pid $=$ S0103-21002011000500010>. Acesso em: 15 maio 2015 .

SILVA, N. F. A Prática da Enfermagem na Bahia: contribuição ao estudo do trabalho dos profissionais de enfermagem de nível superior. Salvador: Gráfica Central, 1987.

SOUZA, C. A.; JERICÓ, M. C.; PERROCA, M. G.

Mapeamento de intervenções/atividades dos enfermeiros em centro quimioterápico: instrumento para avaliação da carga de trabalho. Revista Latino-Americana de Enfermagem, Ribeirão Preto, v. 21, n. 2, mar./abr. 2013. Disponível em: <http://www.revistas.usp.br/rlae/article/view/75949>. Acesso em: 15 maio 2015.

SOUZA, M. K. B. Gestão Compartilhada do SUS municipal no contexto da descentralização: atuação da enfermeira. 2007, 104 f. Dissertação (Mestrado em Enfermagem) - Escola de Enfermagem, Universidade Federal da Bahia, 2007.

TAUBE, S. A. M.; MEIER, J. M. O processo de trabalho da enfermeira na central de material e esterilização. Acta Paulista de Enfermagem, São Paulo, v. 20, n. 4, p. 470-5, 2007. Disponível em: $<$ http://www.scielo.br/scielo.php?pid=S0103$-21002007000400014 \&$ script $=$ sci_abstract\&tlng=pt $>$. Acesso em: 15 maio 2016.

TEIXEIRA, C. F.; VILLASBÔAS, A. L. Q. Modelos de atenção à saúde no SUS: transformação, mudança ou conservação? In: PAIM, J. S.; ALMEIDA FILHO, N. Saúde Coletiva: teoria e prática. Rio de Janeiro: Medbook, 2014, p. 287-302.

TOH, S. G.; ANG, E.; DEVI, M. K. Systematic review on the relationship between the nursing shortage and job satisfaction, stress and burnout levels among nurses in 
oncology/haematology settings. International journal of evidence-based healthcare, Melbourne, v. 10, n. 2, p. 126141, jun. 2012. Disponível em: <https://www.ncbi.nlm. nih.gov/pubmed/22672602>. Acesso em: 15 maio 2016.

URBANETTO, J. S.; CAPELLA, B. B. Processo de trabalho em enfermagem: gerenciamento das relações interpessoais. Revista Brasileira de Enfermagem, Brasília, DF, v. 57, n. 4, p. 447-452, jul./ago. 2004. Disponível em: <http://www.scielo.br/scielo.php?pid=S0034$-71672004000400012 \&$ script $=$ sci_abstract\&tlng=pt $>$. Acesso em: 15 maio 2016.

VARGAS, A. O.; RAMOS, F. R. S. Autonomia na unidade de terapia intensiva: comecemos por cuidar de nós. Revista Brasileira de Enfermagem, Brasília, DF, v. 63, n. 6, p. 956-963, nov./dez. 2010. Disponível em: <http://www.
scielo.br/scielo.php?script=sci_abstract\&pid=S0034$-71672010000600014 \& \operatorname{lng}=$ pt\&nrm=iso $>$. Acesso em: 15 maio 2016.

VEIGA, K. C. G.; FERNANDES, J. D.; PAIVA, M. S. Estudo estrutural das representações sociais do trabalho noturno das enfermeiras. Texto Contexto Enfermagem, Florianópolis, v. 20, n. 4, p. 682-690, out./dez. 2011. Disponível em: <http:// www.scielo.br/scielo.php?script=sci_arttext\&pid $=$ S0104-07072011000400006 $>$. Acesso em: 15 maio 2015

Recebido para publicação em outubro de 2016

Versão final em fevereiro de 2017

Conflito de interesses: inexistente

Suporte financeiro: não houve 